\title{
Revisitando a Função de Reação Fiscal no Brasil Pós- Real: Uma Abordagem de Mudanças de Regime
}

\author{
- Mário jorge Cardoso de Mendonça* - Cláudio hamilton matos dos Santos ** \\ - ADOLFO SACHSIDA***
}

\begin{abstract}
RESUMO
Esse artigo estima a "função de reação fiscal" do setor público consolidado brasileiro após o Plano Real. Para lidar com a incerteza referente às possíveis mudanças de regime ocorridas nesse período, adotou-se o modelo de "Markov-Switching", no qual a probabilidade de mudança de regime é determinada endogenamente. Os resultados obtidos sugerem fortemente que a política fiscal no Brasil apresentou dois regimes distintos após o Plano Real, e o final do ano 2000 marca o período mais provável da transição entre esses dois regimes. O regime "pós-2000" caracteriza-se por uma baixa (ou mesmo nula) reação do superávit primário a variações na dívida líquida do setor público (DLSP). Em contraste, no regime anterior a 2000 (de maior volatilidade) a reação do superávit primário a variações na DLSP é bastante evidente. Observouse, ainda, que em ambos os regimes o superávit primário parece responder positivamente a variações no produto e que em nenhum dos dois regimes o governo parece ter utilizado explicitamente a política fiscal como instrumento de controle da inflação.
\end{abstract}

Palavras-Chave

função de reação fiscal, mudança de regime, modelo de Markov-switching, sustentabilidade da dívida e meta fiscal

\section{ABSTRACT}

This article estimates the "fiscal reaction function" of the Brazilian consolidated public sector after the 1994 "Real Plan". In order to overcome the uncertainty over the dates at which changes in parameters occurred this paper uses the "Markov-switching" model in which the transition probability among regimes is endogenously determined. The results reported here strongly suggest that a major structural break has happened in the Brazilian fiscal policy around the year 2000. Inded, while the "reaction" of primary balance to changes in net debt appears to be weak or even null when one looks at the 2000-2007 period, the contrary happens in the (more volatile) 1995-2000 years. Our results also suggest that the primary surplus is positively correlated with output and uncorrelated with inflation in both regimes.

\section{KEYWORDS}

fiscal reaction function, regime change, Markov-switching model, debt sustainability and fiscal target

\section{JEL ClassificATION}

H6, C22

\footnotetext{
* IPEA - Instituto de Pesquisa Econômica Aplicada. E-mail: mario.mendonca@ipea.gov.br.

** IPEA - Instituto de Pesquisa Econômica Aplicada. E-mail: claudio.santos@ipea.gov.br.

*** IPEA - Instituto de Pesquisa Econômica Aplicada e UCB - Universidade Católica de Brasília. E-mail: sachsida@ hotmail.com.

Endereço para contato: IPEA - Instituto de Pesquisa Econômica Aplicada - Av. Antônio Carlos, 5I/I006. Rio de Janeiro. - RJ. CEP: 20020-0I0.

(Recebido em fevereiro de 2008. Aceito para publicação em março de 2009).
} 


\section{$1 \quad$ INTRODUÇÃO}

A análise da política monetária com a ajuda da chamada "função de reação do Banco Central" tem sido objeto de extensa pesquisa na literatura econômica (TAYLOR, 1993; CLARIDA et al., 2000; SIMS, 1999, 2001). Ainda que relativamente menor, uma literatura análoga surgiu com a preocupação de estimar a "função de reação fiscal" dos Tesouros Nacionais (BOHN, 1998; TAYLOR, 2000; GALI; PEROTTI, 2003; THAMS, 2007).

A correta estimação da função de reação fiscal é importante para a análise da política macroeconômica por pelo menos dois motivos. $\mathrm{O}$ primeiro diz respeito à sustentabilidade da dívida pública. Neste caso, o que se quer saber é se o superávit primário reage a variações na razão dívida pública/PIB (BOHN, op. cit.). Em segundo lugar, a estimação da função de reação fiscal permite investigar se a política fiscal persegue algum outro objetivo além da sustentabilidade da dívida pública - como, por exemplo, a sustentação da demanda agregada em moldes keynesianos tradicionais e/ou o auxílio à Autoridade Monetária no controle da inflação.

No caso brasileiro, vários artigos analisaram o tema da sustentabilidade da dívida líquida do setor público (doravante apenas DLSP), a maioria dos quais aplicando alguma combinação dos testes de Hakkio e Rush (1991), Bohn (1991, 1998) a amostras e dados (conceitualmente e com frequências) bastante diferentes entre si. Não obstante a heterogeneidade dos dados utilizados, a maioria desses estudos (ROCHA, 1997; ISSLER; LIMA, 2000; BICALHO, 2005) chega essencialmente às mesmas conclusões: (i) a DLSP brasileira é sustentável no longo prazo ${ }^{1}$; e (ii) o ajustamento de longo prazo das contas fiscais brasileiras tem se caracterizado por um regime de "spend and tax", isto é, o governo tenderia a gastar sempre que pudesse e a aumentar suas receitas sempre que necessitasse evitar uma explosão de seu endividamento. ${ }^{2}$

Um problema potencialmente sério com a literatura citada no parágrafo anterior identificado também por Simonassi (2007) - é o fato de as técnicas de cointegração utilizadas nesses estudos não serem robustas à existência de quebras estruturais nos dados. Mello (2007) reconhece essas dificuldades e se propõe a lidar com as mesmas trabalhando com diferentes subamostras. Seus resultados mostram variações importantes nos parâmetros relevantes, notadamente um enfraquecimento da reação do superávit primário em relação à DLSP a partir do ano de 2002.

1 Ver Luporini (2000) para uma opinião divergente. A sustentabilidade da DLSP foi também defendida em estudos (menos formais) de economistas formalmente ligados ao Banco Central do Brasil quando da publicação dos mesmos (e.g. GOLDFAJN, 2002; GOLDFAJN; GUARDIA, 2003).

2 A ideia do termo "spend and tax" é salientar uma política fiscal que aumenta primeiro os gastos para depois aumentar os impostos. Ou seja, uma política fiscal em que o aumento do gasto público precede um aumento de impostos. 
O principal objetivo deste artigo é estimar a função de reação fiscal do setor público consolidado, com dados mensais, para o período 1995-2007. A inovação deste estudo reside no uso de procedimentos econométricos capazes de lidar explicitamente com os problemas de quebra estrutural. Propomo-nos a lidar com as referidas quebras estruturais através da estimação de modelos de função de reação fiscal que admitem explicitamente a existência de vários regimes nos dados (denominados "MarkovSwitching Models"), estimando as "probabilidades de transição" entre esses diferentes regimes endogenamente através do uso das chamadas "Cadeias de Markov" (ver HAMILTON, 1989, 1994; KROLZIG, 1997; SIMS, 1999, 2001). Nesse sentido, o "espírito" do presente estudo alinha-se com as contribuições de Garcia e Rigobon (2004), Lima et al. (2006) e Simonassi (op. cit.), ainda que a metodologia utilizada aqui seja bastante distinta das propostas por esses autores. ${ }^{3}$

Antecipando nossas conclusões, notamos que os resultados obtidos com a estimação de modelos Markov-switching (MS) parecem indicar que a política fiscal no Brasil apresentou dois momentos/regimes distintos após o Plano Real. O regime "pós2000" caracteriza-se por uma baixa reação do superávit primário a variações na DLSP. Em contraste, no regime anterior a 2000 a reação do superávit primário a variações na DLSP é bastante evidente. Observou-se, ainda, que em ambos os regimes o superávit primário parece responder positivamente a variações no produto. Além disso, em nenhum dos dois regimes o governo parece ter utilizado explicitamente a política fiscal como instrumento de controle da inflação.

O restante desse estudo está dividido em quatro partes. A seção 2 apresenta uma análise dos fatos estilizados referentes ao comportamento da dívida líquida e do superávit primário do setor público consolidado após o Plano Real. A seção 3 faz uma descrição dos modelos Markov-switching em geral e das especificações utilizadas nesse trabalho em particular. Os resultados obtidos com essas últimas são, então, apresentados e defendidos na seção 4 . Por fim, resumimos nossas principais conclusões na seção 5 .

\section{DEFINIÇOEES, DADOS E FATOS ESTILIZADOS ACERCA DA DÍVIDA PÚ- BLICA NO BRASIL PÓS-REAL}

Iniciamos por relembrar ao leitor alguns fatos incontroversos sobre a dinâmica da dívida líquida do setor público (DLSP) brasileiro. Começando pelas definições mais básicas, notamos que a identidade que descreve a dinâmica da DLSP é dada pela identidade (1) abaixo:

3 Por outro lado, Rocha e Pichetti (2003) utilizam a metodologia de modelos de Markov-Switching para tratar de questões conceitualmente diferentes das que nos propomos a tratar aqui. 


$$
D L S P \equiv D L S P_{-1}+D N+A P(\text { ou } \triangle D L S P \equiv D N+A P)
$$

ou seja, a DLSP no final de um dado "período contábil" é dada pelo valor da mesma no final do período anterior (DLSP.-1) somado ao "déficit nominal" $(D N)$ do setor público e aos "ajustes patrimoniais" $(A P)$ ocorridos no período. Assim sendo, a definição precisa da dinâmica da DLSP implica a necessidade de se definir precisamente dois outros conceitos, i.e., $D N$ e $A P$.

Começando pelo conceito de "déficit nominal" $(D N)$, também conhecido como "necessidades nominais de financiamento do setor público" (ou NFSP nominais ${ }^{4}$ ), notamos que o mesmo é dado pela soma do "déficit primário" $(D P)$ com as despesas líquidas com o pagamento de juros sobre dívida pública (Jur), ou seja,

$$
D N \equiv N F S P \text { nominais } \equiv D P+\text { Jur }
$$

onde "déficit primário do setor público" $(D P)$ é definido na identidade (3) abaixo como o saldo das despesas e receitas "primárias" desse último, ou seja, o valor total das "despesas públicas correntes" excluindo aquelas velativas ao pagamento de juros sobre a dívida pública menos o valor total das receitas públicas "primárias" (ou seja, o valor total das "receitas públicas correntes" excluindo aquelas relativas ao recebimento de juros sobre os haveres públicos). Naturalmente, as despesas e receitas com juros excluídas do cálculo do déficit primário são precisamente as que entram no cálculo de Jur.

$$
D P \equiv N F S P \text { primárias } \equiv \text { Desp Prim - Rec Prim }
$$

Passando agora aos "ajustes patrimoniais" $(A P)$, i.e., às mudanças na DLSP que nada têm a ver com o tamanho relativo das despesas e receitas "correntes" (tanto "primárias" como "com juros") do setor público, cumpre notar que esses podem ser divididos em três grandes grupos: (i) ajustes "cambiais"; (ii) ajustes "relativos às privatizações”; e (iii) "ajustes devidos ao reconhecimento de dívidas” (ou, no jargão, "esqueletos"). O item "i”" reflete as mudanças no valor (em R\$) da DLSP (denominada em moeda estrangeira ou indexada à taxa de câmbio) causadas por variações cambiais. O item "ii" refere-se aos recursos obtidos pelo governo com a venda de suas empresas ao setor privado. Por fim, o item "iiii" são as "novas dívidas" criadas por conta de decisões judiciais e/ou políticas. Longe de serem apenas "detalhes técnicos”, os ajustes patrimoniais são frequentemente determinantes cruciais da dinâmica da dívida pública (ver Gráfico l).

4 Do inglês "public sector borrowing requirements".

5 Mas, curiosamente, incluindo as "despesas de investimento" (em capital fixo), ainda que essas sejam formalmente "despesas de capital" e não "despesas correntes". Ver Blanchard e Giavazzi (2004) para mais detalhes. 
Uma simples análise do Gráfico l parece mostrar que a dinâmica da DLSP é bastante desigual ao longo do tempo, caracterizando assim a existência de diferentes "fases" ou "regimes". Além disso, a DLSP parece estar sujeita a um sem-número de "choques", muitos dos quais gerando "observações aberrantes". Exemplos de "outliers" são os meses de janeiro/fevereiro de 1999 e junho/setembro de 2002 - durante os quais a DLSP aumentou respectivamente R $\$ 100$ bilhões e R 180 bilhões (ou cerca de $20 \%$ em termos reais em ambos os casos) - e abril de 2003, no qual o ajuste cambial diminuiu a DLSP em R $\$ 46$ bilhões (ou cerca de $5 \%$ em termos reais).

GRÁFICO 1

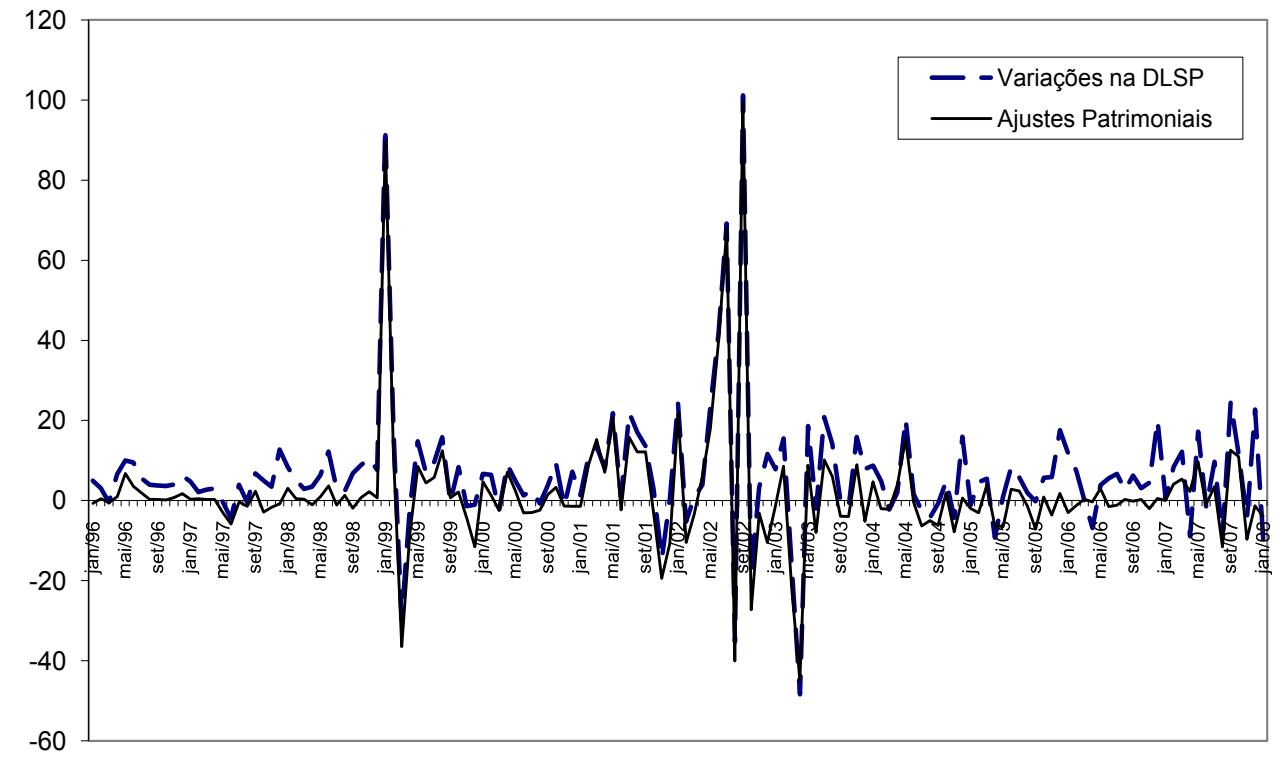

Fonte: Banco Central do Brasil.

Cumpre notar que - embora os ajustes por conta do reconhecimento de dívidas e privatizações também tenham sido significativos no período - os ajustes cambiais foram os principais responsáveis pelas maiores alterações dos ajustes patrimoniais (AP). O Gráfico 2 mostra com mais clareza a importância assumida pela oscilação cambial. 


\section{GRÁFICO 2}

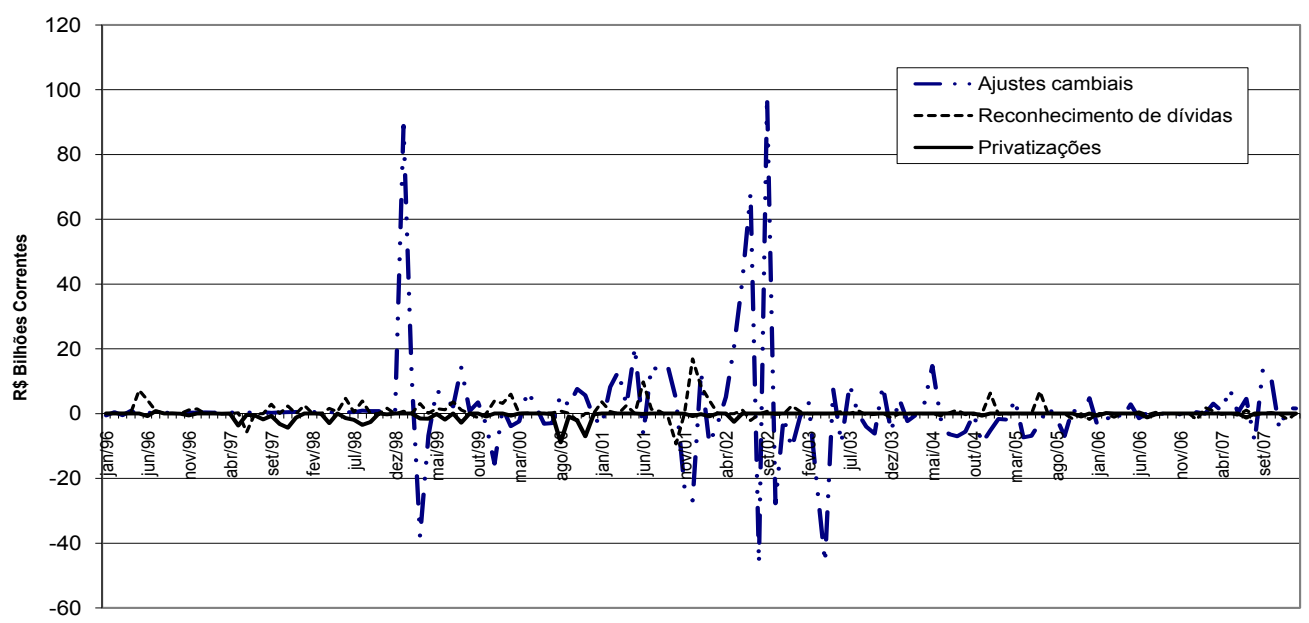

Fonte: Banco Central do Brasil.

\section{METODOLOGIA}

O estudo de modelos econométricos de séries temporais não lineares tem ganhado crescente importância em períodos recentes (KIM; NELSON, 1999; FRANSES; VAN DIJK, 2000; LUTKEPOHL; KRATZIG, 2004). Quando uma relação linear é submetida a uma quebra estrutural - o que pode ocorrer nos coeficientes das variáveis, no intercepto e também na variância dessa relação - os parâmetros do modelo variam com o tempo, resultando em não-linearidades e, via de regra, em violaçôes das hipóteses de estacionariedade e normalidade dos erros dos modelos convencionais.

Uma alternativa é tratar as quebras estruturais (e, portanto, as "mudanças de regime") como exógenas, com a introdução de variáveis dummy em modelos lineares convencionais. Contudo, tal procedimento exige que se conheça antecipadamente o momento exato em que ocorreram as quebras, o que raramente é o caso na prática. E mesmo no caso improvável de o pesquisador "acertar" a data exata da(s) quebra(s) relevante(s) bem como os períodos de duração das quebras, a mera introdução de dummies não resolve problemas relacionados a mudanças de regime na variância dos erros do modelo. Como aponta Sims (2001), é um equívoco grave ignorar essas últimas ou quaisquer outras fontes de não-normalidade nos resíduos ao mesmo tempo em que se leva em conta mudanças nos parâmetros das variáveis. 
Modelos de Markov-switching (MS) (HAMILTON, 1989, 1994; KROLZIG, op. cit.; SIMS, 1999, 2001) caracterizam-se por assumir explicitamente a possibilidade de que, a cada momento do tempo, um número finito (e geralmente pequeno) de "regimes" ou "estados" pode ocorrer, sem que se saiba ao certo qual deles está sendo observado. Apenas para citar um exemplo intuitivo, parece razoável supor que uma economia em recessão se comporte de modo (ou tenha parâmetros) diferente(s) de uma economia em rápido crescimento. Nesse caso, poder-se-ia pensar em dois "regimes", i.e., um "recessivo" e outro "de crescimento", com características bastante diferentes entre si e que se alternam de tempos em tempos, sem que se tenha certeza sobre qual está ocorrendo em cada período específico. Convém frisar, portanto, que modelos MS não assumem que as "mudanças de estado" - por exemplo, a passagem do regime "de crescimento" para o regime "recessivo" - sejam eventos determinísticos. A hipótese é que existem "probabilidades de transição" de um regime para o outro, probabilidades essas estimadas endogenamente pelos modelos MS. ${ }^{6} \mathrm{Nada}$ impede ainda que as mudanças de regime sejam do tipo "once-and-for-all-shifts", em que, após a mudança, determinado regime permanece indefinidamente.

A análise econométrica da função de reação fiscal tem por objetivo testar a hipótese de que o superávit primário se ajusta em resposta às mudanças na dívida pública de modo a assegurar sua sustentabilidade. Além disso, também é possível verificar se a política fiscal está sendo utilizada como instrumento de estabilização do produto ou da inflação. Seguindo a literatura empírica sobre o tema (BOHN, 1998; GALI; PEROTTI, op. cit.; THAMS, op. cit.), propomo-nos a estimar a função de reação fiscal por meio de um modelo Markov-switching que assume a seguinte especificação:

$$
\begin{aligned}
& N F S P_{t}=a\left(s_{t}\right)+\sum_{m=1}^{P} b_{1 m}\left(s_{t}\right) N F S P_{t-m}+\sum_{m=1}^{P} b_{2 m}\left(s_{t}\right) D L S P_{t-m}+\sum_{m=1}^{P} b_{3 m}\left(s_{t}\right) P I N D_{t-m}+ \\
& +\sum_{m=1}^{p} b_{4 m}\left(s_{t}\right) I N F L A_{t-m}+\varepsilon_{t}
\end{aligned}
$$

$\operatorname{com} \varepsilon_{t} \sim N\left(0, \sigma^{2}\left(s_{t}\right)\right)$

onde $N F S P_{t}$ é a necessidade de financiamento primária do setor público consolidado no período $t ; D L S P_{t}$ é a dívida líquida do setor público consolidado no período $t$ medida como proporção do PIB; $P I N D_{t}$ é o produto industrial ${ }^{7}$ no período $t ; \mathrm{e}$ $I N F L A_{t}$ é a inflação no período $t$; e $s_{t}$ é uma variável estocástica não observada que determina o estado $k$ que o modelo assume a cada período $t$.

6 Mais tecnicamente, modelos MS enquadram-se naquilo que Chib (1996) denomina "bidden Markov chain models". Uma ampla variedade desses modelos é apresentada em Kim e Nelson (op. cit.).

7 O produto industrial é usado como proxy para o PIB mensal. 
Note-se que, por hipótese, a "variável latente" $s_{t}$ é regida por um processo estocástico conhecido como uma cadeia de Markov ergódica e definido por uma matriz de probabilidades de transição cujos elementos são dados por:

$$
\begin{aligned}
& p_{i j}=\operatorname{Pr}\left(s_{t+1}=j \mid s_{t}=i\right), \quad \sum_{j=1}^{k} p_{i j}=1 \quad \vee i, j \in\{1, \ldots, k\} \\
& p_{i j} \geq 0 \text { para } i, j=1,2, \ldots, K
\end{aligned}
$$

Aqui, $p_{i j}$ representa a probabilidade de que, em $t+1$, a cadeia mude do regime $i$ para o regime $j$. A ideia, portanto, é que a probabilidade de ocorrência de um regime $s_{t}$ qualquer no presente depende apenas do regime que ocorreu no período anterior, i.e., de $\boldsymbol{s}_{t-1}$. Com $k$ regimes existentes, as probabilidades de transição entre estados podem assim ser representadas pela matriz de transição de probabilidade $\mathrm{P}$, com dimensão $(k \times k)$.

Os parâmetros do modelo acima são estimados a partir da maximização da função de verossimilhança do modelo por meio do algoritmo EM (DEMPSTER; LAIRD; RUBIN, 1977) - uma técnica iterativa para modelos com variáveis omitidas e/ou não observadas. Pode ser mostrado que o valor da função verossimilhança relevante aumenta a cada iteração desse processo, o que garante que o resultado final seja suficientemente próximo do valor máximo da verossimilhança na vizinhança relevante. ${ }^{8}$ É necessário ter em mente, entretanto, que a função de verossimilhança de um modelo MS não possui máximo global (HAMILTON, 1991, 1994; KOOP, 2003). Felizmente, a utilização do algoritmo EM frequentemente leva à obtenção de um máximo local "razoável", com casos patológicos sendo relativamente raros (HAMILTON, 1994).

\section{ANÁLISE EMPÍRICA DA FUNÇÃO DE REAÇÃO FISCAL NO BRASIL}

\subsection{Fontes e Descrição Gráfica dos Dados}

Os dados mensais referem-se ao período de janeiro de 1995 a dezembro de 2007. As variáveis usadas neste estudo (Gráfico 3) são descritas da seguinte forma: ${ }^{9}$

8 Em geral, esse método mostra-se robusto quando os valores iniciais são arbitrados de maneira arbitrária ou pouco eficiente.

9 Os dados podem ser obtidos mediante contato direto com os autores. 
NFSP : valor da necessidade de financiamento primária do setor público consolidado sem valorização cambial acumulado nos últimos 12 meses dividido pelo PIB (também acumulado dessa forma). Fonte: Banco Central do Brasil;

$D L S P$ : razão entre o valor mensal da dívida líquida do setor público consolidado e o PIB (acumulado nos últimos 12 meses e valorizado pelo IGP-DI). Fonte: Banco Central do Brasil;

INFLA : taxa anualizada de inflação medida pelo IPCA em $t{ }^{10}$ Fonte: IBGE;

PIND : taxa anualizada de crescimento do produto industrial em $t,{ }^{11}$ proxy para o crescimento anualizado do PIB real. ${ }^{12}$ Fonte: IBGE.

10 Essa taxa é calculada subtraindo-se 1 da razão entre a soma dos IPCAs entre os períodos $t$ e $t$-12 e a soma dos IPCAs entre os período $t-1$ a $t$-13 e multiplicando esse resultado por 100.

11 Essa taxa é calculada subtraindo-se 1 da razão entre a soma dos índices do quantum da produção industrial entre os períodos $t$ e $t$-12 e a soma desses índices entre os período $t$-l a $t$ - 13 e multiplicando esse resultado por 100.

12 Naturalmente, poderíamos ter usado também a série de PIB mensal disponibilizada pelo Banco Central (anualizada e valorizada pelo IGP-DI e depois deflacionada por esse último índice). Optamos por não fazer isso por dois motivos básicos, quais sejam: (i) os resultados obtidos com essa última série foram qualitativamente similares aos obtidos com a variável PIND; e (ii) não temos conhecimento dos procedimentos precisos de interpolação utilizados pelo Banco Central no cálculo dessa última série a partir do dado oficial trimestral disponibilizado pelo IBGE. 


\section{GRÁFICO 3 - VARIÁVEIS UTILIZADAS NAS REGRESSÕES ECONOMÉTRICAS}
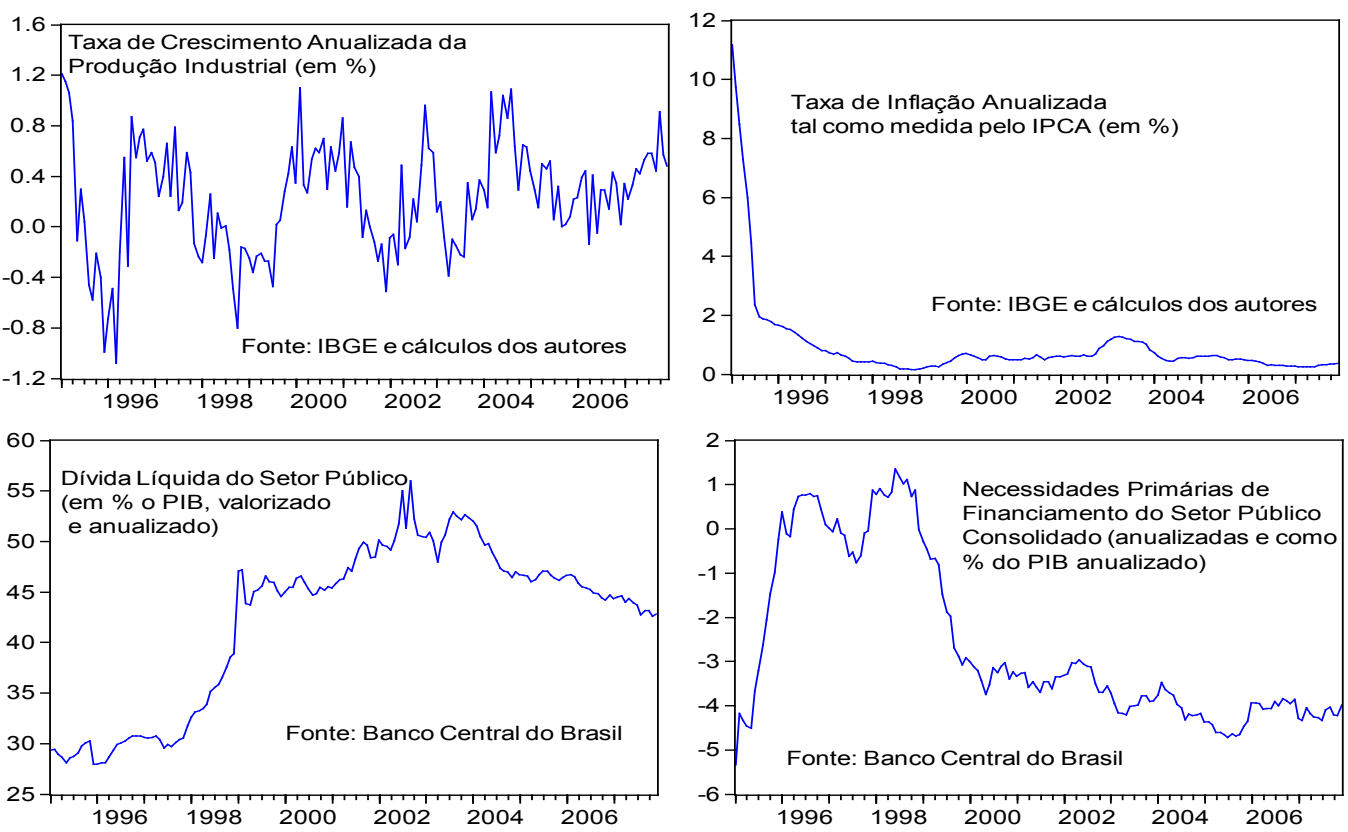

\subsection{Forma Funcional}

Conforme mencionado anteriormente, a função de reação fiscal a ser estimada neste estudo assume o seguinte formato:

$$
N F S P_{t}=b_{0}\left(S_{t}\right)+b_{1}\left(S_{t}\right) N F S P_{t-1}+b_{2}\left(S_{t}\right) D L S P_{t-1}+b_{3}\left(S_{t}\right) I N F L A_{t-1}+b_{4}\left(S_{t}\right) P I N D_{t-1}+\sigma\left(S_{t}\right) \varepsilon_{t}
$$

Alguns comentários se fazem necessários antes de apresentarmos os resultados econométricos que obtivemos com base na equação (6). ${ }^{13}$ Primeiramente, é esperado que exista uma relação negativa entre a NFSP e a dívida pública (i.e, que $b_{2}$ fosse negativo), visto que quando a dívida aumenta é prudente aumentar o superávit primário (e, portanto reduzir a NFSP ${ }^{14}$ ) a fim de garantir uma trajetória sustentável para o endividamento. Em segundo lugar, espera-se que o parâmetro da inflação $\left(b_{3}\right)$ seja negativo no caso de o Tesouro atuar em cooperação com a Autoridade Monetária - já que, nesse caso, um aumento da inflação induziria o Tesouro a gerar

13 A descrição feita neste parágrafo quanto aos sinais esperados para os coeficientes da função fiscal de reação está em concordância com a literatura sobre este tema $(\mathrm{BOHN}, 1998$; TAYLOR, op. cit.; GALI; PEROTTI, op. cit; THAMS, op. cit.

14 Note-se que a NFSP é o superávit primário com sinal trocado. 
um superávit maior (NFSP menor). Em terceiro lugar, notamos que uma política fiscal anticíclica (voluntária ou não) implica um sinal negativo para o parâmetro do produto $\left(b_{4}\right)$ - i.e., diminuições/aumentos no produto induziriam aumentos/diminuições nas NFSP. Por fim, notamos que a inclusão do valor defasado da variável dependente entre as variáveis explicativas tem o intuito de captar algum elemento de inércia na série suavizada da NFSP que deve perder sua força no caso de a economia experimentar saltos e/ou mudanças bruscas de ambiente.

No que se refere à forma funcional, a especificação adotada para a função fiscal de reação expressa na equação (6) é do tipo "backward-looking”. Gali e Perotti (op. cit.), diferentemente, utilizam uma especificação forward-looking introduzindo a expectativa do hiato do produto com uma das variáveis explicativas do modelo. $\mathrm{O}$ fato de não contemplarmos variáveis expectacionais no modelo não nos causa maior incômodo na medida em que, para um modelo univariado, é possível demonstrar que uma especificação do tipo "forward-looking" possui uma forma equivalente do tipo "backward-looking" (SIMS, 1999, 2001).

Cumpre destacar que a estimação da função de reação, com variáveis tomadas em nível, é defensável mesmo na presença de raiz unitária nas variáveis do modelo. ${ }^{15}$ Esse fato é importante no nosso caso porque a aplicação de diversos testes nas séries de dados mostra que as variáveis NFSP e DLSP parecem apresentar raiz unitária. ${ }^{16}$ Felizmente, o problema da "regressão espúria" pode ser contornado por meio da introdução dos valores defasados das variáveis $\mathrm{I}(\mathrm{l})$ entre as variáveis explicativas da regressão (HAMILTON, 1994; SIMS et al., 1990). Com efeito, Hamilton (ibidem, p. 561-562) assinala que tal procedimento assegura uma estimação consistente para o modelo sem mudanças de regime. ${ }^{17}$ Ademais, pode ser demonstrado que, nesse último caso, as estatísticas " $t$ ” para os coeficientes individuais são assintoticamente normais. Assim sendo, devemos incluir os valores defasados de ambas as variáveis NFSP e DLSP entre as variáveis explicativas do nosso modelo (a fim de evitar com-

15 Mais especificamente quando elas são integradas de ordem um.

16 Podemos rejeitar a hipótese de estacionaridade para várias especificaçôes do teste "KPSS", enquanto não podemos rejeitar a hipótese de raiz unitária para diversas especificações dos testes "ADF-t" e de "Perron". Naturalmente, tais resultados (que podem ser obtidos por meio de contato direto com os autores) devem ser vistos com cuidado na presença de quebras estruturais.

17 Hamilton (1994, p. 561-562) aponta que existem três maneiras para a solução do problema da regressão espúria. O primeiro modo se dá a partir da inclusão de valores defasados das variáveis dependentes e independentes numa regressão por OLS, tal como é feito nesse trabalho. O segundo método consiste em rodar a regressão com as variáveis na primeira diferença caso elas sejam em nível I(l). Contudo, deve-se ter em mente que esse procedimento é válido somente caso as variáveis sejam não cointegradas. No caso de as variáveis serem cointegradas, a regressão deve conter o termo de correção de erros (ENGLE; GRANGER, 1987). Por fim, uma terceira maneira é estimar a equação com variáveis em nível com o ajuste de Cochrane-Orcutt para a autocorrelação de primeira ordem nos resíduos. Blough (1992) mostrou que a regressão de Cochrane-Orcutt por método GLS (Generalized Least Squares) é equivalente a uma regressão OLS obtida por variáveis em diferenças. É importante lembrar que se as variáveis forem estacionárias isso pode conduzir a uma regressão mal especificada. 
plicações relacionadas a regressões espúrias). ${ }^{18}$ Por fim, notamos que um argumento adicional contra uma ênfase excessiva na ordem de integração das variáveis em questão é o fato de as variáveis NFSP e DLSP terem sofrido fortes quebras de regime ao longo período amostral (ver seção 2), de modo a (potencialmente, pelo menos) impor considerável viés aos resultados dos testes de raiz unitária convencionais. ${ }^{19}$

\subsection{Resultados Econométricos}

Nesta seção apresentamos os resultados do modelo Markov-Switching (MS) seguindo o procedimento descrito na seção 3. Os resultados obtidos sugerem fortemente que a política fiscal no Brasil apresentou dois regimes distintos após o Plano Real, e o final do ano de 2000 marca o período mais provável da transição entre esses dois regimes. ${ }^{20} \mathrm{O}$ regime "pós-2000" caracteriza-se por uma baixa (ou mesmo nula) reação do superávit primário a variações na dívida líquida do setor público (DLSP). Em contraste, no regime anterior a 2000 (de maior volatilidade) a reação do superávit primário a variações na DLSP é bastante evidente. Na Tabela 1 são apresentadas as estimativas dos parâmetros com os t-valores ${ }^{21}$ da equação (6) para uma especificação MSIAH do modelo, ${ }^{22}$ i.e., para uma especificação que permite mudança no intercepto $(\mathrm{I})$, nos parâmetros das variáveis $(\mathrm{A})$ e na variância $(\mathrm{H})$ em cada regime. Com efeito, a aplicação do teste de razão de verossimilhança (LR) rejeita a hipótese nula

18 Isso também é válido no caso multivariado. De acordo com Sims, Stock e Watson (1990) mesmo na presença de raiz unitária se o objetivo é estimar os parâmetros do VAR não existe necessidade de estimar o VAR nas diferenças. Isto também aparece explicitamente dito em Hamilton (1994, p. 553 e $557)$ e Lutkepohl $(2005$, p. 289). As estatísticas $t$ para checar a significância dos coeficientes além do teste de Wald para, por exemplo, acessar o número de lags da regressão são perfeitamente apropriadas quando a regressão é estimada nos níveis. Um exemplo do que não pode ser implementado quando o VAR é estimado nos níveis diz respeito ao teste de Granger.

19 Além disso, o teste de Saikkonen e Lutkepohl (2002) que permite modelar as observaçóes aberrantes de forma endógena e o teste de Zivot e Andrews (1992) que permite modelar a quebra na tendência e no intercepto também de forma endógena foram aplicados. Seus resultados confirmam a não rejeição da hipótese nula de raiz unitária para a DLSP enquanto ele é não conclusivo para a variável NFSP. Esses testes ainda não indicam a presença de raiz unitária para as outras variáveis do modelo. Os resultados podem ser obtidos com os autores.

20 A introdução de um número maior de regimes gera problemas na rotina numérica do algoritmo EM fazendo com que a matriz de transição de probabilidade se torne não ergódica. A hipótese de ergocidade é uma das principais suposições do modelo.

21 As estatísticas de erro padrão foram obtidas computacionalmente por procedimento numérico. Infelizmente, essas aproximações não são necessariamente precisas.

22 Essa terminologia é adotada por Krolzig (op. cit.). 
de linearidade $\left(\mathrm{LR}=44.31, X_{(6)}^{2}=[0.000]^{* *}\right.$ e $\left.X_{(8)}^{2}=[0.000]^{* *}\right),{ }^{23}$ ratificando a escolha pelo modelo MS(2)IAH(1 $)^{24}$ para a base de dados analisada.

Como se percebe da análise da Tabela 1 e também pelo Gráfico Al do Apêndice 1 (notadamente da trajetória da probabilidade suavizada ${ }^{25}$ ), existe uma clara diferença na condução do superávit primário antes e depois do ano de $2000 .{ }^{26}$ Esse resultado parece-nos perfeitamente compatível com a ideia de que a introdução do regime de metas fiscais representou uma mudança do tipo "once and for all" na política fiscal brasileira. Notamos, adicionalmente, que o regime 1 (pré-2000) se mostra mais instável do que o regime 2 (pós-2000) - com efeito, a volatilidade medida pelo desvio padrão do regime 1 é o dobro da volatilidade do regime 2 (ver a sétima linha da Tabela 1).

TABELA 1 - MODELO MS(2)-AIH(I)* - VARIÁVEL DEPENDENTE: NFSP

\begin{tabular}{lcc}
\hline & Regime 1 & Regime 2 \\
\hline Const & $1.3353(4.567)$ & $-0.2002(-0.4531)$ \\
NFSP(-1) & $0.8529(22.3511)$ & $0.9251(23.9239)$ \\
DLSP(-1) & $-0.0389(-4.8349)$ & $-0.0015(-0.1798)$ \\
INFLA(-1) & $-0.0402(-1.246)$ & $-0.0094(-0.1044)$ \\
PIND(-1) & $-0.1917(-2.2808)$ & $-0.0706(-1.3055)$ \\
Desvio Padrão & 0.30499 & 0.15167 \\
Observações & 65 & 90 \\
Verossimilhança & & 23.79 \\
\hline
\end{tabular}

Nota: ${ }^{*}$ t-valor entre parênteses.

De maneira geral, os resultados presentes na Tabela 1 indicam que no regime 1 a autoridade fiscal aparentemente se vê obrigada a reagir às mudanças na DLSP (já que esta variável possui coeficiente negativo e significativo) - enquanto no regime

$23 \mathrm{O}$ teste LR posto aqui possui distribuição não padronizada, não podendo ser caracterizado analiticamente desde que as probabilidades de transição são não identificadas sob a hipótese de linearidade. Contudo, é possível mostrar que essa distribuição pode ser aproximada se situando no intervalo entre duas distribuições qui-quadrado. Deduz-se disso que se para ambas as distribuições a hipótese nula for rejeitada, o teste LR deverá necessariamente fazê-lo. Contrariamente, se não houver rejeição da hipótese nula de linearidade em ambos os casos, então o mesmo deverá acontecer para o teste LR. Em qualquer outra situação nada poderá ser dito (DAVIES, 1977; HANSEN, 1992).

24 Modelo MS-IAH para dois regimes e uma defasagem.

25 A probabilidade suavizada (smoothed) considera informações de toda amostra, sendo definida da seguinte forma: $\operatorname{Pr}\left[S_{t}=j \mid \Psi_{T}\right]$, onde $\Psi_{T}$ é o conjunto de informação pleno até o instante $T$. A probabilidade filtrada (filtered) é uma inferência ótima no estado da variável no tempo $t$ considerando as informações até $t$, enquanto a probabilidade predita (predicted) considera a informação até $t-1$.

26 Essa conclusão é obtida também quando se utiliza a taxa suavizada de crescimento do PIB real tal como medida pelo Banco Central no lugar da variável PIND. 
2 a dinâmica do superávit é determinada fundamentalmente pela "meta fiscal", não respondendo a variaçóes de curto prazo na DSLP. Notamos, ainda, que em ambos os regimes o superávit parece reagir positivamente ao produto - ou seja, a política fiscal parece ser moderadamente anticíclica - ainda que o coeficiente do produto não seja significativo no regime 2 (o que não nos parece surpreendente, dados os limites impostos pela "meta"). ${ }^{27} \mathrm{E}$, embora o coeficiente defasado da NFSP seja significativo em ambos os regimes, ele é menor no regime 1 - o que sugere um grau de discricionariedade relativamente maior da política fiscal no período pré-2000. Finalmente, notamos que em nenhum dos estados a variável inflação apresentou significância. Deduz-se, assim, que não parece ter existido uma atuação conjunta do Tesouro e do Banco Central para controlar a inflação. ${ }^{28}$ Naturalmente, essa interpretação pode não ser estritamente válida para o regime pós-2000, uma vez que a imposição de metas fiscais rígidas e públicas por si só pode ter ajudado a manter o controle inflacionário.

TABELA 2 - PROBABILIDADES DE TRANSIÇÃO

\begin{tabular}{ccc}
\hline & Regime 2 & Regime 1 \\
\hline Regime 2 & 1.0000 & 0.0000 \\
Regime 1 & 0.01508 & 0.9849 \\
\hline
\end{tabular}

Passando agora à análise da matriz de transição de probabilidades (Tabela 2), notamos que, uma vez dentro de um dos dois regimes, existe uma alta probabilidade de permanência dentro de tal regime. No regime 2, em particular, o retorno ao regime l é muito improvável, o que sugere que a mudança na política fiscal ocorrida após a crise de 1999 foi de caráter estrutural e permanente.

\subsection{Análise de Robustez}

A fim de investigar a robustez dos resultados, obtidos com o modelo MS(2)-IAH(1), devemos compará-los aos resultados obtidos com modelos alternativos. Ficamos tentados, primeiramente, a investigar a possibilidade de que um modelo MS(2)$\mathrm{IH}(\mathrm{l})$ de dois regimes - i.e., um modelo em que apenas o intercepto e a variância

27 Note-se que essa conclusão não se verifica quando se utiliza a taxa suavizada de crescimento do PIB real tal como medida pelo Banco Central (no lugar da variável PIND). Nesse último caso, a resposta da política fiscal ao PIB não é significativa em nenhum dos dois regimes.

28 Note-se que essa conclusão não se verifica quando se utiliza a taxa suavizada de crescimento do PIB real tal como medida pelo Banco Central (no lugar da variável PIND). Nesse último caso, a política fiscal parece responder à inflação no regime l. Essa mesma conclusão é obtida também no modelo com a variável PIND quando se adiciona o segundo "lag" da variável DLSP entre as variáveis explicativas. 
variam em cada regime, enquanto os parâmetros das variáveis explicativas mantêm-se inalterados - pudesse se ajustar melhor aos dados do que o modelo apresentado na seção anterior. A ideia que está por trás do uso de um modelo desse tipo é que não teria havido mudança significativa na condução da política fiscal ao longo de todo o período 1995-2007 (como parece sugerir SIMONASSI, op. cit.). O que fundamentalmente teria ocorrido, nesse caso, seria uma alternância entre momentos de "crise" e "tranquilidade", e nesses últimos a condução da política fiscal seria mais "fácil". 29

A estimação dos parâmetros por meio do modelo $\mathrm{MS}(2)-\mathrm{IH}(1)$ gera valores semelhantes aos encontrados na Tabela 1. Novamente, excetuando-se a inflação, todas as variáveis são significativas e apresentam os sinais esperados. De acordo com esse modelo, a condução da política fiscal teria objetivado a sustentabilidade da dívida e sido anticíclica ao longo de todo o período, sem responder a variações na inflação, resultados esses que comprovam a robustez do modelo original MS(2)-IAH(1). ${ }^{30}$ Apenas para finalizar a análise, gostaríamos de salientar que o teste de AIC sugere que o modelo original $[\mathrm{MS}(2)-\mathrm{IAH}(1)]$ é mais adequado que o modelo alternativo [MS(2)-IH(1)]. Além disso, o valor da verossimilhança para o modelo MS(2)-IAH(1) é superior em quase $40 \%$ em relação ao modelo concorrente. Assim, tendemos a aceitar que MS(2)-IAH(1) é um modelo superior ao modelo alternativo [MS(2)$\mathrm{IH}(1)] .^{31}$

Outro teste de robustez seria estimarmos a função de reação fiscal por um modelo VAR. Após isso, poderíamos aplicar as técnicas usuais de cointegração e fazermos inferência estatística sobre os resultados. Quando toda a amostra é levada em consideração, o procedimento de Johansen (1991) aponta para a existência de cointegração entre as variáveis NFSP, DLSP e produto (PIND). Contudo, tal evidência fica bem mais fraca quando se nota que: (i) os modelos VAR que servem como ponto de partida para a aplicação do procedimento de Johansen apresentam erros não normais e heteroscedásticos; (ii) a cointegração é perdida quando os testes de cointegração mencionados acima são aplicados aos dados do subperíodo 2001-2007 (como, aliás, seria de se supor à luz dos resultados reportados na seção anterior); e (iii) a aplicação do teste de cointegração com quebra estrutural de Gregory e Hansen (1996) aos dados do período 1995-2007 indica a inexistência de cointegração entre as referidas

29 Uma análise desta natureza para o estudo da política monetária para os EUA aparece em Sims (1999, 2001).

30 No que se refere aos parâmetros estimados, ao gráfico da probabilidade suavizada e à matriz de transição para este modelo, observamos que os mesmos são semelhantes aos do modelo MS(2)-AIH(1). Por motivos de economia de espaço não apresentamos essas informações - que podem, entretanto, ser obtidas mediante contato direto com os autores.

31 Todos os passos acima foram replicados para o modelo de duas defasagens sem obtenção de nenhuma mudança significativa nos resultados. Os resultados podem ser obtidos por meio de contato direto com os autores. 
séries - com quebra estimada para agosto de $1997 .{ }^{32}$ Assim sendo, não podemos fazer inferência estatística a partir do VAR estimado neste artigo. Isto fortalece nossa escolha original por um modelo com mudança de regime (Markov-Switching), que é capaz de lidar adequadamente com a presença de quebras estruturais nas séries de dados.

A fim de submeter nossa interpretação dos dados a um último teste de robustez, realizamos a estimação da função de reação fiscal por mínimos quadrados ordinários para dois períodos distintos. A primeira subamostra refere-se ao período de janeiro de 1995 até outubro de 2000 (a data da mudança de regime de acordo com o modelo da Tabela 1), e a segunda subamostra começa a partir de então e vai até o final do período amostral. Os resultados parecem corroborar as conclusões obtidas com o modelo MS(2)-IAH(1) reportado na Tabela $1 .{ }^{33}$

\section{COMENTÁRIOS FINAIS}

Os vários modelos econométricos discutidos neste trabalho parecem dar suporte à visão de que a política fiscal brasileira sofreu um importante ponto de inflexão após a crise cambial de 1999. A adoção do regime de metas explícitas, e relativamente invariantes, para o superávit primário teve como resultado a redução de sua variância. Além disto, ocorreu também uma redução na sensibilidade da resposta do superávit primário a variações de curto prazo na DLSP e do produto.

Naturalmente, isso não significa que a política fiscal tenha deixado de enfatizar a sustentabilidade da DLSP - a elevação da meta para a NFSP em 2003, após a crise cambial de 2002, e a contínua diminuição da DLSP medida como percentagem do PIB ocorrida desde então deixam claro que esse não foi o caso. Com efeito, a experiência brasileira ao longo da primeira década do século 21 parece indicar que a "reação" do superávit primário à DLSP - no sentido econométrico da expressão - não é condição necessária para a sustentabilidade da dívida. A intuição por trás desse fato é simples: metas elevadas, e relativamente fixas, para o superávit primário garantem a sustentabilidade da dívida pública. Esse fato independe da dinâmica de curto prazo dessas variáveis.

De maneira geral, três são as principais conclusões desse estudo:

32 Nenhum desses resultados é apresentado nesse texto, mas estão disponíveis àqueles que quiserem contactar os autores. Os testes Gregory e Hansen foram realizados no software RATS, utilizando, respectivamente, os procedimentos egtest.src e gregoryhansen.src disponíveis gratuitamente em www. estima.com.

33 Por motivos de economia de espaço esses resultados não estão reportados, mas estão disponíveis àqueles que quiserem contatar os autores. 
a) A política fiscal apresentou dois regimes distintos após o Plano Real. Outubro de 2000 é a data mais provável da transição entre esses dois regimes. Além disso, a mudança na política fiscal ocorrida após a crise de 1999 parece ter sido de caráter estrutural e permanente.

b) No regime 1 (vigente até outubro de 2000) a reação do superávit primário a variações na DLSP é bastante evidente. Já o regime 2 (vigente após outubro de 2000) caracteriza-se por uma baixa (ou mesmo nula) reação do superávit primário a variações na dívida líquida do setor público (DLSP).

c) Em ambos os regimes o superávit primário parece responder positivamente a variações no produto. Isso pode caracterizar uma política fiscal anticíclica. Contudo, em nenhum dos dois regimes o governo parece ter utilizado explicitamente a política fiscal como instrumento de controle da inflação.

\section{REFERENCIAS}

AGUIAR, M. T. Dominância fiscal e a regra de reação fiscal: uma análise empírica para o Brasil. 2007. Dissertação (Mestrado) - FEA/USP. São Paulo.

BICALHO, A. Testes de sustentabilidade e ajuste fiscal no Brasil pós-Real. 2005. Dissertação (Mestrado) - EPGE-FGV. Rio de Janeiro.

BLANCHARD, O; GIAVAZZI, F. Improving the stability and growth pact through a proper accounting of public investment. London, Centre for Economic Policy Research, 2004. (Discussion Paper, n. 4220).

BLOUGH, S. Spurious regression with AR(1) correction and unit root pretest. John Hopkins University, 1992. Mimeo.

BOHN, H. The sustainability of budget deficits with lump-sum and with incomebased taxation. Journal of Money, Credit and Banking, v. 23 n. 3, p. 581-604, 1991.

. The behavior of U.S. public debt and deficits. The Quarterly Journal of Economics, 113, p. 949-963, 1998.

CHIB, S. Calculating posterior distributions and modal estimates in Markov mixtures models. Journal of Econometrics, 75, p. 79-97, 1996.

CLARIDA, R.; GALI, J.; GERTLER, M. Monetary policy rule and macroeconomic stability: evidence and some theory. Quarterly Review of Economics, CXV, p.147$180,2000$.

COOK, S. Spurious rejection by cointegration tests incorporating structural change in the cointegrating relationship. Applied Economics Letters, 11, p. 879-884, 2004. 
DAVIES, R.B. Hypothesis testing when a nuisance parameter is present only under the alternative, Biometrika, 64, p.247-254, 1977.

DEMPSTER, A. P.; LAIRD, N. M.; RUBIN, D. B. Maximum likelihood from incomplete data via the EM algorithm. Journal of Royal Statistical Society, 39, p. 1-38, 1977.

ENGLE, R.; GRANGER, C. Cointegration and error correction: representation, estimation and testing. Econometrica, 55, p.251-276, 1987.

FRANSES, P. H.; DIJK, D. V. Non-linear time series models in empirical finance. Cambridge University Press, 2000.

GALI, J.; PEROTTI, R. Fiscal policy and monetary integration in Europe. Economic Policy, v.18, n. 37, p. 533-572, 2003.

GARCIA, M.; RIGOBON, R. A risk management approach to emerging market's sovereign debt sustainability with an application to Brazilian data. NBER Working Paper, n. 10336, 2004.

GIAMBIAGI, F. Do déficit de metas às metas de déficit: a politica fiscal do governo Fernando Henrique Cardoso 1995/2002. Rio de Janeiro: BNDES, 2002 (Texto para Discussão, n. 93).

. A politica fiscal do governo Lula em perspectiva histórica: qual é o limite para o aumento do gasto público? Rio de Janeiro: IPEA, 2006. (Texto para Discussão, n. 1169).

GOLDFAJN, I. Are there reasons to doubt fiscal sustainability in Brazil? Banco Central do Brasil, 2002. (Technical Note, n. 25).

.; GUARDIA, E. R. Fiscal rules and debt sustainability in Brazil. Banco Central do Brasil, 2003. (Technical Note, n. 39).

GRANGER, C. W. J. Strategies for modelling non-linear relationships. Economic Record, v. 69, p. 233-238, 1993.

GREGORY, A. W.; HANSEN, B. E. Residual-based tests for cointegration in models with regime shifts. Journal of Econometrics, v. 70, n. 1, p. 99-126, 1996.

HAKKIO, C.; RUSH, M. Is the budget deficit too large? Economic Inquiry, v. 29, n. 7, p. 429-445, 1991.

HAMILTON, J. D. Time series analysis. Princeton University Press, 1994.

. A Quasi-Bayesian approach to estimating parameters for mixtures of normal distributions. Journal of Business and Economic Statistics, v. 9, n. 1, p. 27-39, 1991.

. A new approach to the economic analysis of nonstationary time series and the business cycle. Econometrica, v. 57, n. 2, p. 357-384, 1989.

; FLAVIN, M. On the limitations of government borrowing: a framework for empirical testing. American Economic Review, v. 76, p. 809-819, Sept. 1986. 
HANSEN, B. E. The likelihood ratio test under nonstandard assumptions: testing the Markov switching model of GDP. Journal of Applied Econometrics, v. 7, p. S61-S82, 1992; erratum v. 11, p. 195-198, 1996.

ISSLER, J.; LIMA R. Public debt sustainability and endogenous seigniorage in Brazil: time series evidence from 1947-92. Journal of Development Economics, v. 62, n. 1, p. 131-147, 2000.

JOHANSEN, S. Estimation and hypothesis testing of cointegration vectors in Gaussian vector autoregressive models. Econometrica, v. 59, p. 1551-1580, 1991.

.; MOSCONI, R.; NIELSEN, B. Cointegration analysis in the presence of structural breaks in the deterministic trend. Econometrics Journal, 3, p. 216-249, 2000.

KIM, C.; NELSON, C. State-space models with regime switching. MIT Press, 1999.

KOOP, G. Bayesian econometrics. John Wiley, 2003.

KROLZIG, H. Markov switching vector autoregressions. Modelling, statistical inference and application to business cycle analysis. Berlin: Springer-Verlag, 1997.

LEYBOURNE, S.; NEWBOLD, P. Spurious rejections by cointegration tests induced by structural breaks. Applied Economics, 35, p. 1117-1121, 2003.

LIMA, L. R.; SAMPAIO, R.; GAGLIANONE, W. Debt ceiling and fiscal sustainability in Brazil: a quantile autoregression approach. Mimeo. Rio de Janeiro: EPGE/ FGV, 2006.

LUPORINI, V. Sustainability of the Brazillian fiscal policy and Central Bank independence. Revista Brasileira de Economia, v. 54, n.2, p. 201-226, 2000.

LUTKEPOHL, H.; KRATZIG, M. Applied time series econometrics. Cambridge: Cambridge University Press, 2004.

MANFRINI, S.; FREIRE, F. Meta de superávit primário fica em 4,25\% do PIB, a maior da história. Folha de São Paulo, 08/02/2003.

MELLO, L. Estimating a fiscal reaction function: the case of debt sustainability in Brazil. Applied Economics, v. 40, p. 271-284, 2007.

$\mathrm{NOH}, \mathrm{J}$; KIM, T. Behaviour of cointegration tests in the presence of structural breaks in variance. Applied Economics Letters, 10, p. 999-1002, 2003.

ROCHA, F. Long-run limits on the Brazilian government debt. Revista Brasileira de Economia, v. 4, p. 447-470, 1997.

.; PICHETTI, P. Fiscal adjustment in Brazil. Revista Brasileira de Economia, v. 57, n. 1, p. 239-252, jan/mar 2003.

SIMONASSI, A. Função de resposta fiscal, múltiplas quebras estruturais e a sustentabilidade da dívida pública no Brasil. XXXV ENCONTRO DA ANPEC, Recife-PE, 2007.

SIMS, C. Hidden Markov Chain models. Lecture notes. Princeton University, 2005. 
. Stability and instability in US monetary policy behavior. Princeton University, 2001. (Discussion Paper).

. Drifts and breaks in monetary policy. Princeton University, 1999. (Discussion Paper).

.; STOCK, J.; WATSON, M. Inference in linear time series models with some unit roots. Econometrica, v. 58, n. 1, p. 161-182, 1990.

TAYLOR, J. B. Discretion versus policy rules in practice. Carnegie-Rochester Conference Series on Public Policy, v. 39, p. 195-214, 1993.

. Reassessing discretionary fiscal policy. Journal of Economic Perspectives, v. 14, p. 21-36, 2000.

THAMS, A. Fiscal policy rules in practice. SFB 649, 2007. (Discussion Paper n.16).

ZIVOT, E.; ANDREWS, D. Further evidence on the great crash, the oil-price shock and the unit-root hypothesis. Journal of Business and Economic Statistics, v. 10, n. 3, p. 251-270, 1992. 


\section{APÊNDICE 1 - OS GRÁFICOS DO MODELO MS(2)-IAH(1)}

FIGURA A.I - PROBABILIDADES SUAVIZADA, FILTRADA E PREVISTA

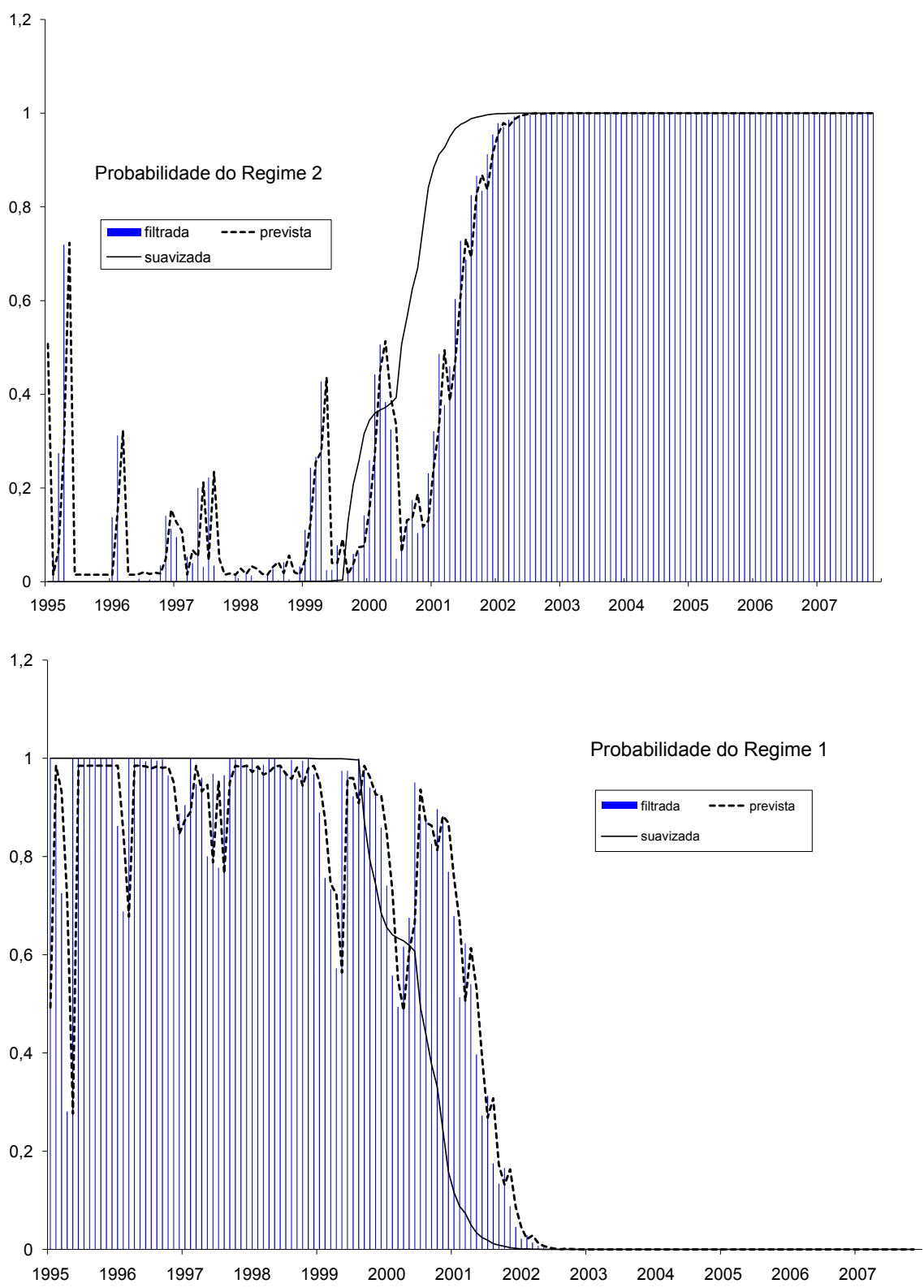




\section{FIGURA A.2 - AJUSTAMENTO E PREVISÃO UM PASSO À FRENTE}

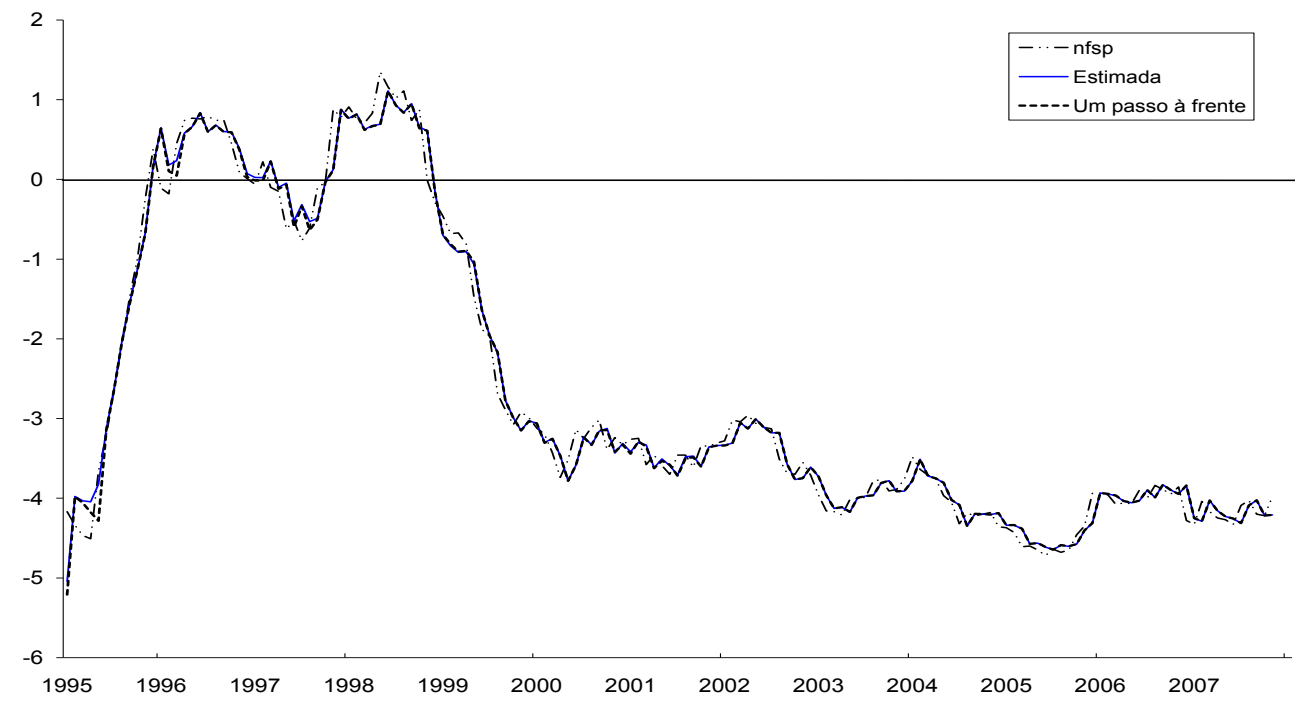

